\title{
A HIGH-PERFORMANCE METHOD FOR SIMULATING SURFACE RAINFALL-RUNOFF DYNAMICS USING PARTICLE SYSTEM
}

\author{
Fangli Zhang ${ }^{\mathrm{a}, \mathrm{b}}$, Qiming Zhou ${ }^{\mathrm{b}}$, Qingquan $\mathrm{Li}^{\mathrm{a}}$, Guofeng $\mathrm{Wu}^{\mathrm{a}}$, Jun $\mathrm{Liu}^{\mathrm{c} *}$ \\ ${ }^{a}$ Key Laboratory for Geo-Environmental Monitoring of Coastal Zone of the National Administration of Surveying, \\ Mapping and GeoInformation \& Shenzhen Key Laboratory of Spatial-temporal Smart Sensing and Services, \\ Shenzhen University, Shenzhen, China - flzhang@life.hkbu.edu.hk, (liqq, guofeng.wu)@ szu.edu.cn \\ ${ }^{\mathrm{b}}$ Department of Geography, Hong Kong Baptist University, Kowloon, Hong Kong, China - qiming@ @ hkbu.edu.hk \\ ${ }^{\mathrm{c}}$ Shenzhen Institute of Advanced Technology, Chinese Academy of Sciences, Shenzhen, China - \\ jun.liu@siat.ac.cn
}

Commission II, WG II/5

KEY WORDS: Hydrologic Model, Rainfall Runoff Process, Flow Path Network, Particle System, Parallel Computing

\begin{abstract}
:
The simulation of rainfall-runoff process is essential for disaster emergency and sustainable development. One common disadvantage of the existing conceptual hydrological models is that they are highly dependent upon specific spatial-temporal contexts. Meanwhile, due to the inter-dependence of adjacent flow paths, it is still difficult for the RS or GIS supported distributed hydrological models to achieve high-performance application in real world applications. As an attempt to improve the performance efficiencies of those models, this study presents a high-performance rainfall-runoff simulating framework based on the flow path network and a separate particle system. The vector-based flow path lines are topologically linked to constrain the movements of independent rain drop particles. A separate particle system, representing surface runoff, is involved to model the precipitation process and simulate surface flow dynamics. The trajectory of each particle is constrained by the flow path network and can be tracked by concurrent processors in a parallel cluster system. The result of speedup experiment shows that the proposed framework can significantly improve the simulating performance just by adding independent processors. By separating the catchment elements and the accumulated water, this study provides an extensible solution for improving the existing distributed hydrological models. Further, a parallel modeling and simulating platform needs to be developed and validate to be applied in monitoring real world hydrologic processes.
\end{abstract}

\section{INTRODUCTION}

Surface runoff, also known as overland flow, is one of most important hydrologic processes in water resource management and studies. Rainfall-runoff simulation is an important technique for detecting and quantifying surface runoff dynamics during the hydrologic processes. The rainfall-runoff simulating models are essential for risk prediction, disaster warning, and sustainable development.

There are essentially two main methods to model and simulate the rainfall-runoff process, the empirically based models and the physically based models. The former, the conceptual or the theoretical hydrological model, also known as the so-called "black-box", uses recorded data to predict surface runoff based on mathematical models such as regression analysis, support vector machine (SVM) (Lin et al., 2006), artificial neural network (ANN) (Wu et al., 2009), adaptive network-based fuzzy inference systems (ANFIS) (Talei et al., 2010) and particle swarm optimization (PSO) algorithm (Chou, 2012). However, the runoff dynamics are susceptible to many parameters such as hydrologic, geologic and topographic factors (Li et al., 2014), the applications of these models are hampered by calibration and adaptable adjustment. The latter, the distributed hydrological model, increasingly supported by geographical information system (GIS) and remote sensing (RS) technology, tries to model the physical movements of water based on structured surface elements. These structured surface elements are created from cell or TIN based digital elevation model (DEM), such as the topography-based hydrological model (TOPMODEL), the Sys-

${ }^{*}$ Corresponding author tem Hydrologic European (SHE) model, and the soil and water assessment tool (SWAT) (Chen et al., 2014). The GIS-supported simulating methods can provide physically dynamic results and adapt to various spatial scales. However, it is still difficult for these raster or vector based models to achieve high simulating efficiency. One important reason is the inter-dependence when iteratively accumulating the surface flows for these linked surface elements.

Chen et al. (2014) point out that the performance efficiencies of existing facet-based methods, whether cell-based or TIN-based, are still inadequate for real-timely modeling and simulating in real world. Therefore, this study proposes a high-performance rainfall-runoff simulation framework based on the vector-based flow path network and a separate particle system. Based on the raster DEM data, significant topographic feature points and main streamlines can be extracted by CPE method (Zhou and Chen, 2011), and then these terrain points and hard lines can be used to create a drainage-constrained TIN (Chen et al., 2014). A set of source points are randomly selected for modeling raindrop sites, and the corresponding flow paths can be traced along the steepest-descent direction over the TIN surface. The trajectory of each particle is constrained by this flow path network and can be tracked by the cluster system of processors in parallel. A rainfall event is simulated by pouring raindrops to different source point sites, and the surface flow dynamics can be simulated by the movements of the independent particles. Experiments are conducted to assess and validate the computing ability of the particle system constrained by the topological flow path network.

Hence, this study aims to explore a parallel simulating framework for real-timely monitoring. At present, however, very limited 
experiments are conducted to preliminarily validate the parallel performance efficiency, and further study may focus on developing such a high-performance simulating platform for real world applications.

\section{RELATED WORK}

Rainfall-runoff is an important hydrologic process in the water cycle. The rainfall-runoff simulation models can quantitatively estimate the surface runoff dynamics during the hydrologic processes.

Previous studies have focused more on conceptually quantifying daily or annual flow accumulation for the entire research region, but less on physically capturing the spatial or temporal variations in hydrological processes. As shown in Table 1, the existing rainfall-runoff models can be categorized into two types: namely the empirically based conceptual models (the so-called "black-box") and the physically based distributed models. By regarding rainfall-runoff process as a simple input-output system, the empirical models estimate the daily or annual surface flow accumulations according to their empirical relationships with the recorded precipitation data. Correspondingly, the physical models, especially the GIS-supported triangle facet network model, can better describe the spatial and temporal variabilities of the hydrological processes. Based on the surface catchment elements, the gird cells, or the flow path lines, these physically based models can provide physically dynamic results and adapt to various spatial scales. However, it is still difficult for these cell or vector based models to achieve high-performance simulation of rainfall-runoff dynamics over large research areas. The main reason is the inter-dependence of flow accumulation on the linked catchment elements during the hydrological processes.

Table 1: The two main kinds of surface runoff models.

\begin{tabular}{cll}
\hline Approach & Description & Author(s) \\
\hline SCS & soil conservation & (USDA, 1972) \\
CREAMS & runoff and erosion & (Knisel, 1980) \\
VIC-2L & variable infiltration & (Abdulla et al., 1997) \\
SVM & support vector & (Lin et al., 2006) \\
ANN & neural network & (Wu et al., 2009) \\
ANFIS & adaptive network & (Talei et al., 2010) \\
PSO & particle swarm & (Chou, 2012) \\
\hline TOPMODEL & topography-based & (Beven et al., 1979) \\
SHE & European distributed & (Abbott et al., 1986) \\
Form & topographic form & (Pilesjö et al., 1998) \\
SWAT & soil and water & (USDA, 1994) \\
HIMS & hydro-informatic & (Liu et al., 2008) \\
TFN & triangulated facet & (Zhou et al., 2011) \\
RunCA & cellular automata & (Shao et al., 2015) \\
\hline
\end{tabular}

\section{METHODOLOGY}

To achieve high-performance simulation efficiency for further development and application, the parallel computing techniques are involved to solve computing tasks by a cluster system of processors concurrently. The TIN-based flow path network model is different from the above cell-based models, it can provide a one dimensional representation for the land surface that adapt to various spatial scales. As constrained by the topologically linked flow path lines, an independent particle system can be used to represent rain drops, while the movements of these particles can model and simulate the rainfall-runoff process in parallel.
The methodology adopted for the simulating framework can be conducted in two major steps (Figure 1):

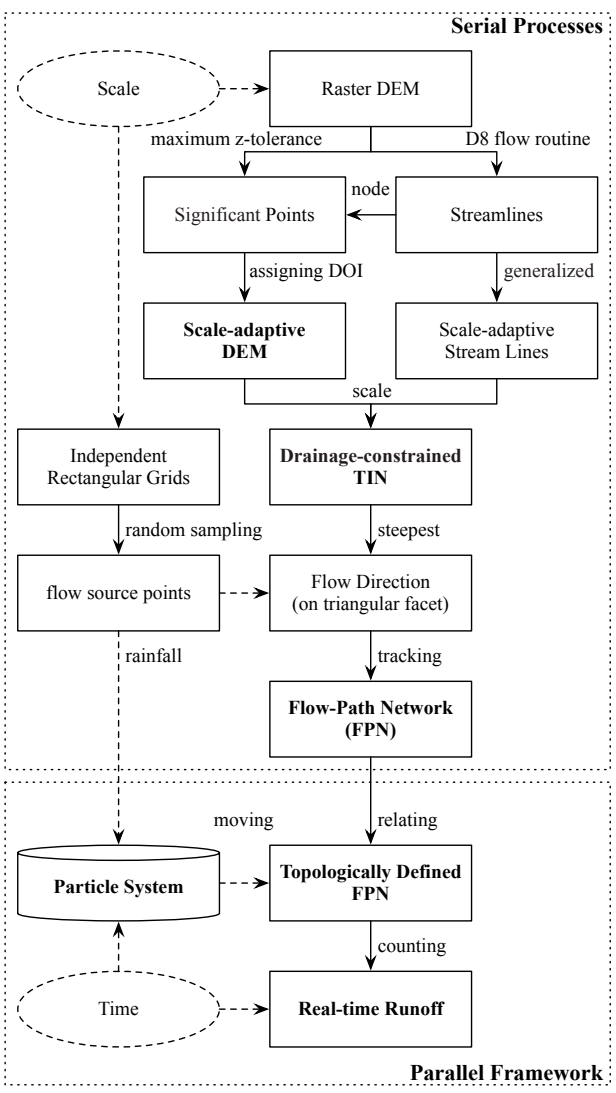

Figure 1: The overall framework for high-performance surface rainfall-runoff modeling and simulating.

(1) Serial processes.

a. Constructing the scale-adaptive DEM from raster DEM;

b. Creating drainage-constrained TIN at the selected scale;

c. Tracing flow paths from random source points over the TIN surface.

(2) Parallel framework.

a. Topologically linking the flow path nodes and lines, calculating the velocities for each flow line;

b. Let the independent particles move along with the flow path network;

c. Tracking and counting the moving water particles for selected regions.

\subsection{Serial Processes}

For surface rainfall-runoff dynamics modeling and simulating, the hydrological variables such as slope, aspect and drainage network may subsequently change with the spatial scale of land surface representations. To ensure consistency and quality of the drainage network which must be adaptive to a given scale, the scale-adaptive DEM (S-DEM) is involved to support a scaleadaptive TIN to match the demand from surface flow simulation at any coarser scale (Chen and Zhou, 2013).

As shown in Figure 1, based on the given scale, the S-DEM is created by significant terrain points and streamlines extracted 
from raster DEM, the D8 algorithm and the Douglas-Peucker algorithm are used to extracting the significant points and the main streamlines (O'Callaghan and Mark, 1984; Douglas and Peucker, 1973), the CPE method is used to integrate the D8 significant points, the maximum z-tolerance points and the D8 main streamlines for supporting the consistency and quality of the S-DEM structure (Zhou and Chen, 2011). Then the S-DEM elevation points at the given scale and the main streamlines are combined to create the drainage-constrained TIN (Chen et al., 2014). Besides, a fishnet grid is created independently and the source points are randomly sampled from each grid cell. According to the steepest-descent rules, the flow path lines can be tracked from each source points to its target catchment area.

\subsection{Parallel Framework}

Based on the flow path network model, as shown in Figure 2, the source points and the flow path lines can be linked according to the topological tables. The node table recorded all the path nodes, and the source points are labeled. The flow paths are splitted into two-node lines, which are recorded and linked by the start node and end node in the line table. Besides, the flow velocity for each line can be calculated according to the Mannings's Equation, which is one of the most commonly used equations for open channel flow.

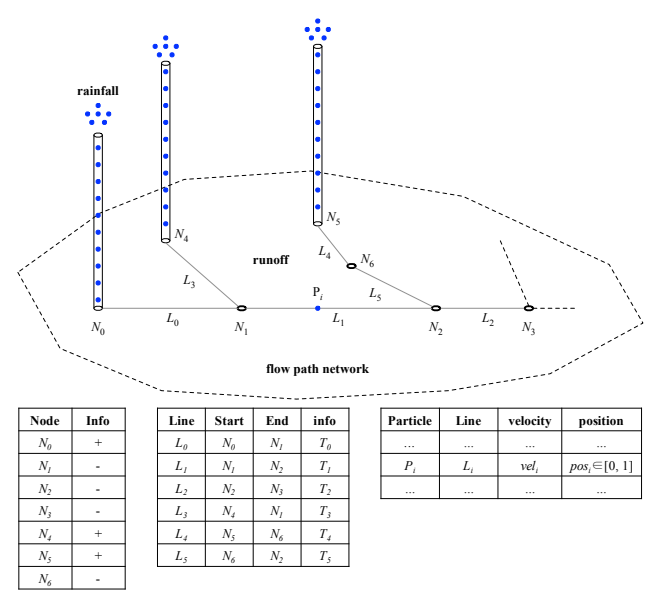

Figure 2: The flow path network and the particle system.

In addition to the above two topological tables, the distribution of the particles can also be recorded and iterated in a table, which records the real-time velocity, the adhering flow line and the relative position for each particles. During the rainfall-runoff process, each particle, representing water, starts to move from the randomly selected source points, and keeps on moving along with the linked flow lines. At a specific interval, the instant velocity and relative position of each particle can be calculated by the following equations:

$$
\begin{aligned}
V_{i}(t) & =V_{L_{i}(t)} \\
L_{i}(t+\Delta t) & =f\left(L_{i}(t), \Delta t, F P N\right) \\
V_{i}(t+\Delta t) & =V_{L_{i}(t+\Delta t)}
\end{aligned}
$$

where $\quad i=$ any selected moving particle

$t, \Delta t=$ any given time point and a time interval

$V_{i}(t)=$ the moving velocity of particle $i$ at time $t$

$L_{i}(t)=$ the adhering flow line of particle $i$ at time $t$

$V_{L_{i}}=$ the flow velocity of flow line $L_{i}$
Hence, the trajectory of each particle relies only on the source point, form which the specific flow path and velocity can be determined. As constrained by the topologically linked network, the independent movements can be tracked in parallel, therefore, this particle system can provide the potential for high-performance modeling and simulating.

\section{EXPERIMENTS AND DISCUSSIONS}

At present, only some limited experiments are conducted to explore and validate the computing ability of this parallel framework. As shown in Figure 3, the particle system are parallelized for computing tests. For each core of each processor in the cluster system, the entire flow path network is loaded for constraining moving routes. For particle system, rain drops are assigned to different source points at different rates for representing surface flow dynamics.

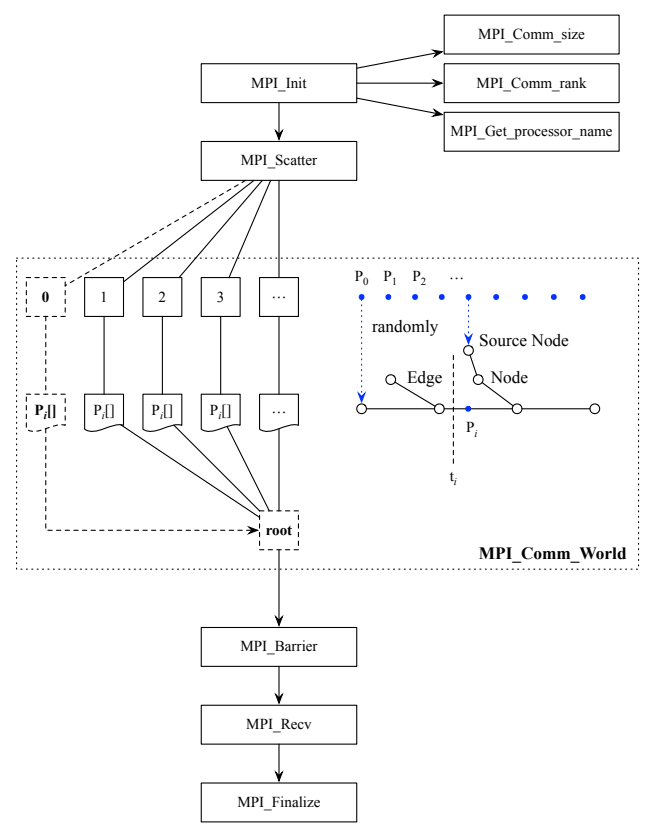

Figure 3: The parallel framework for high-performance simulating rainfall-runoff dynamics based on particle system.

The message passing interface (MPI) is the most widely used standard that can be used to exchange data between processes and processor units (http://www.mpi-forum.org). Based on MPICH framework, a high-performance and widely portable implementation of the MPI standard (https://www.mpich.org), we preliminarily tested the influences of the computing object number and the processing unit number on the computing time.

In the particle test, an increasing number of rain drop particles are iteratively tracked with a certain number (e.g., 4 cores) of cluster CPU cores. It aims to test the influence of computing object quantity on computing time. Likewise, in the processor test, a fixed number (e.g., 10, 000 particles) of rain drop particles are iteratively tracked by an increasing number of CPU cores. It can test the influence of processing unit size on performance efficiency. As shown by Figure 4 (a), apparently, the computing time increases almost linearly as the number of rain drop particles increases. For each iteration, when the particle number increases from 10,000 to 150,000 , the computing time is also about 10 times longer. 


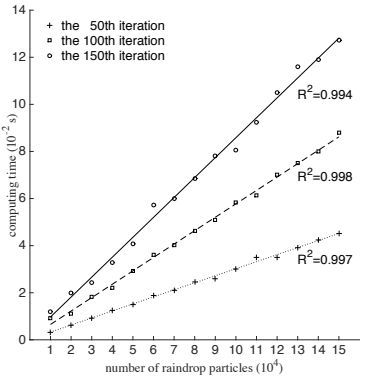

(a) Particle number test

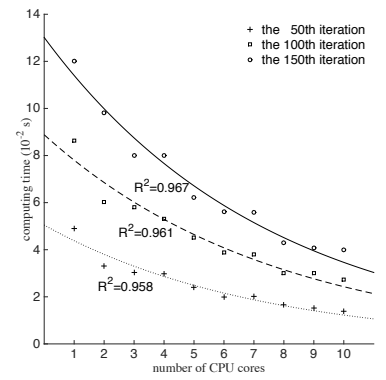

(b) Processor number test
Figure 4: The test results for processor and particle numbers.

Since the rainfall particles are independent and calculated with a time complexity of $O(n)$, which could explain the linear relationships between the computing time and the raindrop number. Besides, as shown in Figure 4 (b), when the particles remain the same, and the number of CPU cores increases from 1 to 10 , the computing time declines approximately exponentially. That is, the increasing communication cost between more and more processors influences the speedup rate of the cluster system. In summary, the computing time can be effectively shorten by adding processing units to the cluster system, hence the proposed parallel framework can provide the potential for highperformance modeling and simulating rainfall-runoff dynamics in real world applications.

\section{CONCLUSIONS}

This study preliminarily explored a parallel framework for highperformance simulating surface rainfall-runoff dynamics over large areas with the assistance of the scale-adaptive flow path network and an independent particle system. Constrained by the topologically linked flow path lines, the movements of independent particles can represent the overland surface flow dynamics. By involving the separate particle system, the surface runoff dynamics can be independently simulated on a parallel cluster system. Though lots of real-world validation experiments are in progress, the results of the linear speedup experiments indicate that this parallel framework can effectively reduce the processing time and improve the performance efficiency by physically increasing the number of processors. Therefore, the combination of the vector flow path network and the parallelizable particle system is capable of providing real-time surface flow dynamics in large regions.

\section{ACKNOWLEDGEMENTS}

This work is jointly supported by the National Natural Science Foundation of China (No. 41471340 and No. 41301403), the Research Grants Council (RGC) of Hong Kong General Research Fund (GRF) (Project No. 203913), the Chongqing Basic and Advanced Research General Project (No. cstc2013jcyjA40010), and the Hong Kong Baptist University Faculty Research Grant (No. FRG2/14-15/073).

\section{REFERENCES}

Abbott, M. B., Bathurst, J. C., Cunge, J. A., \& OConnell, P. E., 1986. An introduction to the European Hydrological SystemSysteme Hydrologique Europeen, SHE, 1: History and philosophy of a physically-based, distributed modelling system. Journal of Hydrology, 87(1-2), pp. 4559.
Abdulla, F. A. \& Lettenmaier, D. P., 1997. Development of regional parameter esti- mation equations for a macroscale hydrologic model. Journal of Hydrology, 197(1-4), pp. 230257.

Beven, K. J. \& Kirkby, M. J., 1979. A physically based, variable contributing area model of basin hydrology / Un modele a base physique de zone dappel variable de lhydrologie du bassin versant. Hydrological Sciences Bulletin, 24(1), pp. 4369.

Chen, Y. \& Zhou, Q., 2013. A scale-adaptive DEM for multiscale terrain analysis. International Journal of Geographical Information Science, 27(7), pp. 1329-1348.

Chen, Y., Zhou, Q., Li, S., Meng, F., Bi, X., Wilson, J. P., Xing, Z., Qi, J., Li, Q. \& Zhang, C., 2014. The simulation of surface flow dynamics using a flow-path network model. International Journal of Geographical Information Science, 28(11), pp. 22422260 .

Chou, C. M., 2012. Particle Swarm Optimization for Identifying Rainfall-Runoff Relationships. Journal of Water Resource and Protection, 04(03), pp. 115-126.

Douglas, D.H. \& Peucker, T.K., 1973. Algorithms for the reduction of the number of points required to represent adigitized line or its caricature. Cartographica: the International Journal for Geographic Information and Geovisualization, 10 (2), pp. 112122.

Knisel, W. G, 1980. CREAMS: A field-scale model for chemicals, runoff and erosion from agricultural management systems. Technical report.

Li, H. Y., Sivapalan, M., Tian, F. \& Harman, C., 2014. Functional approach to exploring climatic and landscape controls of runoff generation: 1. Behavioral constraints on runoff volume. Water Resources Research, 50(12), pp. 9300-9322.

Lin, J., Cheng, C. \& Chau, W. K., 2006. Using support vector machines for long-term discharge prediction. Hydrological Sciences Journal, 51(4), pp. 599-612.

O'Callaghan, J. F. \& Mark, D. M., 1984. The extraction of drainage networks from digital elevation data. Computer Vision, Graphics, and Image Processing, 28(3), pp. 323-344.

Pilesjö, P. \& Hasan, A., 2014. A Triangular Form-based Multiple Flow Algorithm to Estimate Overland Flow Distribution and Accumulation on a Digital Elevation Model. Transactions in GIS, 18(1), pp. 108-124.

Shao, Q., Weatherley, D., Huang, L., \& Baumgartl, T., 2015. RunCA: A cellular au- tomata model for simulating surface runoff at different scales. Journal of Hydrology, 529, pp. 816829.

Talei, A., Chua, L. H. C. \& Wong, T. S. W., 2010. Evaluation of rainfall and discharge inputs used by Adaptive Network-based Fuzzy Inference Systems (ANFIS) in rainfallrunoff modeling. Journal of Hydrology, 391(3-4), pp. 248-262.

Wu, C. L., Chau, K. W. \& Li, Y. S., 2009. Predicting monthly streamflow using data-driven models coupled with data preprocessing techniques. Water Resources Research, 45(8), W08432.

Zhou, Q. \& Chen, Y., 2011. Generalization of DEM for terrain analysis using a compound method. ISPRS Journal of Photogrammetry and Remote Sensing, 66(1), pp. 38-45.

Zhou, Q., Pilesjö, P. \& Chen, Y., 2011. Estimating surface flow paths on a digital elevation model using a triangular facet network. Water Resources Research, 47, W07522. 